Anaesthesist 2011 · 60:101-102 DOI 10.1007/s00101-011-1853-8

Online publiziert: 12. Februar 2011

(c) Springer-Verlag 2011

\section{J.U. Bleyl}

Klinik für Anästhesiologie und Intensivtherapie, Carl Gustav Carus Universitätsklinikum, Dresden

\title{
Vitale Grenzsituationen gemeinsam gemeistert
}

Der in der vorliegenden Ausgabe von Der Anaesthesist publizierte Übersichtsbeitrag „Perioperatives anästhesiologisches Management bei ausgedehnten Leberteilresektionen. Pathophysiologie der Lebererkrankungen und funktionelle Zeichen des Leberversagens" erlaubt einen Blick zurück auf die gemeinsamen anästhesiologischen und chirurgischen Erfolge bei Leberresektionen, aber auch eine kritische Bestandsaufnahme des Status quo [1]. Leberresektionen waren noch vor nicht allzu langer Zeit mit Grenzsituationen verbunden, die es gemeinsam, chirurgisch und anästhesiologisch, zu meistern galt. Heute ist die Leberresektion ein etabliertes operatives Verfahren zur Therapie maligner Prozesse in der Leber. Gründe für diese Entwicklung sind ein verbessertes Verständnis für die Physiologie und Pathophysiologie der Leber, eine Verbesserung der operativen Techniken und eine Optimierung der perioperativen anästhesiologischen Versorgung von Patienten. Diese Fortschritte haben zu einem Rückgang der perioperativen Letalität von über 30\% auf 2-3\% geführt und in etablierten Zentren Leberresektionen zu einem Routineeingriff werden lassen $[2,3]$.

Chirurgische Kollegen bestätigen, dass die Reduktion der perioperativen Letalität bei Leberresektionen in hohem Maß einem verbesserten intraoperativen anästhesiologischen Management zuzuschreiben ist [3]. Cochrane-Analysen aus dem Jahr 2009, die sich mit unterschiedlichen Aspekten des intraoperativen anästhesiologischen Managements bei Leberresektionen beschäftigen, hinterlassen jedoch auch einige Fragezeichen hinter anschei- nend wohl etablierten Bestandteilen dieser Strategie.

Ein zentraler Bestandteil des intraoperativen Konzepts bei Leberresektion ist es, durch einen niedrigen zentralen Venendruck (ZVD) den Druck in den Lebervenen zu senken und damit die Blutungen aus den hepatischen Sinusoiden bei der Resektion zu reduzieren. So zeigen Studien, dass ein intraoperativer ZVD $<5 \mathrm{mmHg}$ mit einem geringeren Blutverlust und einer geringeren Transfusionspflichtigkeit sowie einem deutlich besseren postoperativen „outcome“ einhergeht $[4,5]$. Eine aktuelle Cochrane-Analyse, in die 9 Studien mit 587 Patienten eingingen, konnte die Reduktion des Blutverlusts durch Senkung des ZVD allerdings nicht bestätigen [6]. Die geringe Patientenzahl und die Heterogenität der untersuchten Studien lassen eine abschließende Empfehlung zurzeit nicht zu. Auch die Autoren der Analyse haben diese Problematik erkannt und verlangen eine Bestätigung ihrer Ergebnisse durch prospektiv randomisierte Studien. Einen differenzierteren Ansatz bietet eine neue Studie, die die Messung des ZVD an der Höhe des operativen Eingriffsorts ausrichtet und so eine realistischere Angabe über den definitiven hepatischen Venendruck auf Resektionsniveau erlaubt [7]. Klinische Ergebnisse zum Effekt dieser Methode stehen zum gegenwärtigen Zeitpunkt jedoch aus.

Blutverlust und Transfusionsbedarf werden in der Literatur als unabhängige Faktoren für eine erhöhte postoperative Morbidität und Letalität nach Leberresektionen beschrieben [5]. Zudem werden Bluttransfusionen mit einer erneuten Manifestation von hepatozellulären Kar- zinomen in Verbindung gebracht. Verantwortlich für diese Effekte sind wahrscheinlich Spenderleukozyten, die trotz aufwendiger Separationsverfahren in Erythrozytenkonzentraten (EK) verbleiben und nach Transfusion immunmodulatorisch wirken. Trotz Einführung leukozytendepletierter EK in Europa zu Beginn dieses Jahrzehnts gibt es bislang keine Studie, die die Auswirkung der Depletion auf die Morbidität, die Letalität und die Tumorremanifestation bei Leberresektionen untersucht hat. Pharmakologische Ansätze, durch intraoperative Gabe von Aprotinin (in Deutschland nicht mehr zugelassen) oder Tranexamsäure die Blutung zu minimieren und die Transfusionspflichtigkeit zu reduzieren, werden durch eine Cochrane-Analyse unterstützt [8]. Allerdings ließ sich kein Einfluss dieser Maßnahmen auf die perioperative Morbidität und Letalität nachweisen.

Die präoperative Gabe von Steroiden bei Leberresektionen mit vollständiger vaskulärer Okklusion wird durch die Ergebnisse der Cochrane-Analyse unter gewissen Vorbehalten unterstützt [9]. Die Steroidgabe hat einen positiven Einfluss auf den Ischämie-Reperfusion-Schaden und ist mit einer reduzierten Freisetzung proinflammatorischer Zytokine und geringeren postoperativen Bilirubinspiegeln verbunden [10]. Dies ist von besonderem Belang, gilt doch der postoperative Bilirubinspiegel als ein hervorragender Parameter in der Beurteilung der Leberfunktion nach Leberresektion.

Das anästhesiologische Management bei Leberresektionen steht auch unter Einbeziehung der Cochrane-Analysen auf einem soliden Fundament, bedarf aber prospektiv randomisierter Studien, um 
die bestehenden Unsicherheiten zu beseitigen und neue Verfahren evidenzbasiert zu etablieren.

Die chirurgischen Fortschritte der letzten Jahre, die v. a. auf einer optimierten, CT-gestützten Operationsplanung, auf einer Weiterentwicklung und Verfeinerung der Operationsverfahren sowie auf einer Entwicklung von leberschonenden Resektionsinstrumentarien (Ultraschall, Wasserstrahl, Ultrazision) beruhen, haben ohne Zweifel zu einer Reduktion der perioperativen Morbidität und Letalität geführt [11]. Die klinischen Erfolge bei der Leberresektion beruhen somit auf einem Puzzle einzelner, sehr verschiedener Faktoren aus unterschiedlichen medizinischen Bereichen. Ganz entscheidend für den Erfolg war und ist jedoch der enge Kontakt von Anästhesist und Chirurg, der Blick vom Anästhesisten und vom Chirurgen über das Tuch! Die Leberresektion ist ein erfolgreich gelebtes Beispiel für den Leitgedanken unseres diesjährigen Jahreskongresses, „Vitale Grenzsituationen gemeinsam meistern“, zum Wohl unsere Patienten.

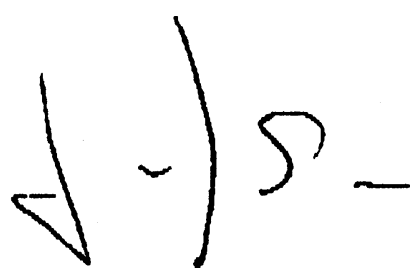

J.-U. Bleyl

\section{Korrespondenzadresse}

\section{PD. Dr. J.U. Bleyl}

Klinik für Anästhesiologie und Intensivtherapie,

Carl Gustav Carus Universitätsklinikum

Fetscherstraße 74, 01307 Dresden

Joerg.Bleyl@uniklinikum-dresden.de
4. Jones RM, Moulton CE, Hardy KJ (1998) Central venous pressure and its effect on blood loss during liver resection. Br J Surg 85:1058-1060

5. Hofland J, Henny CP (2007) Bloodless (liver) surgery? The anesthetist's view. Dig Surg 24:265-273

6. Gurusamy KS, Li J, Sharma D, Davidson BR (2009) Cardiopulmonary interventions to decrease blood loss and blood transfusion requirements for liver resection. Cochrane Database Syst Rev:CD007338

7. Giordano C, Deitte LA, Gravenstein N, Rice MJ (2010) What is the preferred central venous pressure zero reference for hepatic resection? Anesth Analg 111:660-664

8. Gurusamy KS, Li J, Sharma D, Davidson BR (2009) Pharmacological interventions to decrease blood loss and blood transfusion requirements for liver resection. Cochrane Database Syst Rev:CD008085

9. Abu-Amara M, Gurusamy KS, Hori S et al (2009) Pharmacological interventions versus no pharmacological intervention for ischaemia reperfusion injury in liver resection surgery performed under vascular control. Cochrane Database Syst Rev: CD007472

10. Hayashi Y, Takayama T, Yamazaki S et al (2011) Validation of perioperative steroids administration in liver resection: a randomized controlled trial. Ann Surg 253:50-55

11. Rahbari NN, Mehrabi A, Mollberg NM et al (2011) Hepatocellular carcinoma: current management and perspectives for the future. Ann Surg, doi 10.1097/SLA.0b013e31820d944f

\section{Forschungspreisausschreibun- gen der DGIIN 2011}

Die Deutsche Gesellschaft für Internistische Intensivmedizin und Notfallmedizin (DGIIN) schreibt

1. den Forschungspreis „Intensivmedizin“ (dotiert mit 5.000 Euro)

und

2. den Forschungspreis "Notfallmedizin" (dotiert mit 2500 Euro)

für das Jahr 2011 aus.

Die Forschungspreise dienen der Auszeichnung besonderer wissenschaftlicher experimenteller und klinischer Arbeiten auf dem Gebiet der internistischen und allgemeinen Intensivmedizin bzw. der Notfallmedizin von Mitgliedern der Deutschen Gesellschaft für Internistische Intensivmedizin und Notfallmedizin. Die weiteren Kriterien sind in den Vergaberichtlinien (siehe www.dgiin.de) zu ersehen.

Die Bewerber sind gebeten, die Preisarbeit entsprechend den Vergaberichtlinien bis zum 15. März 2011 bei der Geschäftsstelle der DGIIN einzureichen (Geschäftsstelle DGIIN e.V., Seumestraße 8, 10245 Berlin).

Die Preisträger werden über die Zuerkennung der Forschungspreise bis spätestens 31. Mai 2011 informiert.

Die Verleihung erfolgt anlässlich der Jahrestagung der Deutschen Gesellschaft für Internistische Intensivmedizin und Notfallmedizin und der Österreichischen Gesellschaft für Internistische und Allgemeine Intensivmedizin vom 15.-18. Juni 2011 in Wien. Eine Kostenübernahme für die Teilnahme am Kongress durch die DGIIN kann für die Preisträger nicht erfolgen.

\section{Literatur}

1. Herz S, Puhl, Spiess C et al (2011) Perioperatives anästhesiologisches Management bei ausgedehnten Leberteilresektionen. Pathophysiologie der Lebererkrankungen und funktionelle Zeichen des Leberversagens. Anaesthesist 60:103-117

2. Foster JH (1991) History of liver surgery. Arch Surg 126:381-387

3. Kamiyama T, Nakanishi K, Yokoo H et al (2010) Perioperative management of hepatic resection toward zero mortality and morbidity: analysis of 793 consecutive cases in a single institution. J Am Coll Surg 211:443-449 